\title{
Berichte aus den Landesgruppen
}

\section{Burgenland}

Krankheitsbedingt fand 2011 nur ein Kurs statt: 10.06.-11.06.2011, Hüftsonografiekurs Grundkurs

\section{A. K. Klabuschnigg}

\section{Niederösterreich}

$\nabla$

Folgende Aktivitäten fanden in der Landesgruppe NÖ im Jahr 2011 statt: Ultraschallkurs, geburtshilfliche und gynäkologische Ultraschall-Tipps und Tricks für die tägliche Praxis mit hands-on Übungen am 18.6.2011.

Treffen der ÖGUM Stufe 2-Schaller am 24.11.2011 mit Referat: Neues aus der fetalen Neurosonografie, Referent Prim.Dr. Anzböck

Anzböck

\section{Salzburg \\ $\nabla$}

Vorsitz: Dr. Erich Hübner, Wahlarzt für Radiologie, Haydnstrasse 1, 5020 Salzburg

Stellvertreter: OA Dr. Christian Weismann, SALK, St. Johanns Spital, Universitätsinstitut für Radiodiagnostik, Sondervertrag für Mammadiagnostik \& Mammaintervention

Die Aktivitäten der Salzburger ÖGUMSektion sind auch im Jahr 2010/2011 traditionell durch zahlreiche Kursveranstaltungen gekennzeichnet. Ich darf mich an dieser Stelle erneut sehr herzlich bei den Organisatoren und Referenten dieser ÖGUM-Kurse für die geleistete Fortbildungsarbeit bedanken.

Seit Ende 2009 ist nun die Landesgruppe auch im Internet unter www.oegum-salzburg.at vertreten. Die Seite befindet sich im Aufbau und soll in Zukunft über Kursaktivitäten und Fortbildungen informieren.

Die Landesgruppe Salzburg veranstaltet unter der Leitung des neuen Kursleiters OA. Hollerweger in Zusammenarbeit mit dem KH der BHB Ultraschallkurse seit März 2010. Ziel dieser Kurse ist es, in Kleingruppen den Teilnehmern eine qualitativ hochwertige Ausbildung zu bieten. Die nachfolgende Liste (redaktionell gekürzt), geordnet nach den jeweiligen
Fachbereichen, bietet einen Überblick über die Kurstätigkeit im Jahr 2011 der Salzburger ÖGUM-Sektion:

- 5 Abdomen-Kurse von OA. Dr. Alois Hollerweger, Prim. Univ. Prof. Dr. N. Gritzmann

- 5 Kardiologie-Kurse von OÄ Dr. B. Maurer-Dietze

- 3 Mamma-Kurse von OA Dr. Christian Weismann

Für das Jahr 2012 sind von den ÖGUMKursleitern der Sektion Salzburg bereits zahlreiche Kurse geplant.

\section{E. Hübner}

\section{Steiermark}

$\nabla$

Kurse 2011 abgehalten: Sonografie der Säuglingshüfte, Pädiatrische Ultraschalldiagnostik je 1 Grund- und Aufbaukurs. G. Schweintzger

\section{Tirol}

\section{$\nabla$}

Als Leiterin der Landesgruppe Tirol darf ich über 3 Kurse informieren, die 2011 abgehalten wurden:

- 19 Mai 2011 Kontrastmittelsonografie: Abendveranstaltung Innsbruck

- 1-2 April: Workshop Sono, Knie und Fuß, Innsbruck

- 15. April 2011: Abdomensonografie Grundkurs, Kursleiter Prim. Univ. Prof. Dr. Kathrein

\section{Andrea S Klauser}

\section{Wien}

$\nabla$

Vorstand der Landesgruppe Wien (Wiederwahl am 2.12.2011):

> Vorsitzender: Prof. Dr. Wolfgang Eppel

- Sekretär: Prof. Dr. Dieter Bettelheim

- Kassier: Prof. Dr. Wolfgang Dock 5. Mitglied: Ass. Prof. Dr. Christian Kollmann

Jahreshauptversammlung am 2.12.11 AKH Wien, Hörsaalzentrum

\section{Abgehaltene Kurse der}

Landesgruppe Wien:

- Angiologie: Carotis/Abschlußkurs, 14.-15.1.2011 in Salzburg (Doz. Katzenschlager)

- Abdomen: Grundkurs 22.-23.1.2011 (Ass. Prof. Brichta) 
- Gynäkologie:Gyn Allround, 24.2.3.3.2011, Ägypten (Prof. Deutinger)

- Kopf/Hals: Carotis und Schilddrüse, 18.-19.2.2011 (Prof. Gessl)

- Kopf/Hals: Schilddrüse, 12.3.2011 (Doz. Zettinig)

- Gynäkologie: Ultraschall konkret, 1.2.4.2011 (Prof. Hafner)

- Pädiatrie: Neonatologischer Ultraschall, 29.-30.4.2011 (Dr. Zoder)

- Gynäkologie: Sun \& Sound, Mediterranes Fortbildungsseminar, 28.5.4.6.2011, Ibiza (Prof. Deutinger)

- Abdomen: Grundkurs, 30.5.-3.6.2011, Ärztetage in Grado (Prof. Mostbeck)

- Pädiatrie: Schädelsonografie, Grundkurs, 30.9.-1.10.2011 (Dr. Zoder)

- Pädiatrie: Schädelsonografie, Aufbaukurs, 3.10.2011, Graz (Dr. Zoder)

- Abdomen: Grundkurs, 21.-22.10.2011, Baden (Prof. Gritzmann)

- Angiologie: Farbdopplersonografie Halsgefäße und periphere Gefäße, 20.22.10.2011 (Prof. Gritzmann)

- Bewegungsapparat: 2nd Interdisciplinary Musculosceletal Ultrasound Course Vienna, 21.-22.10.2011, Innsbruck (Priv. Doz. Schüller-Weidekamm)

- Gynäkologie: Mediterranes Fortbildungsseminar, „hormonell-aktuell“, 24.-31.10.2011, Lanzarote (Prof. Deutinger)

- Abdomen/Kontrastmittel: Kontrastmittelsonografie, 4.11.11 (OA. Aiginger)

- Pädiatrie: Hüftsonografie, 11.12.11.2011 (Dr. Zoder)

- Abdomen: Grundkurs, 18.-20.11.2011 (OA. Aiginger)

\section{Fortbildungen der}

Landesgruppe Wien:

Interdisziplinäre Fortbildung am 2.12.2011 im AKH Wien, Hörsaalzentrum, verbunden mit der Jahreshauptversammlung der Landesgruppe Wien, organisiert und moderiert von Ass. Prof. Brichta ( $\mathrm{H}$. Prosch/Wien: Sonografie der supraclaviculären Lymphknoten in der Abklärung thorakaler Erkrankungen; G. Bodner/ Wien: Ultraschallgezielte Interventionen an peripheren Nervenpathologien; $M$. Riccabona/Graz: Sonografie des (früh) kindlichen Abdomens - mehr als nur die Niere?! Kindertypische Beispiele aus dem klinischen Alltag)

\section{A. Brichta}

Von den Landesgruppen Oberösterreich, Kärnten und Vorarlberg lagen bis Redaktionsschluß leider keine Berichte vor. 